Research Paper

\title{
Effect of Platelet-Rich Plasma on Ischemia-Reperfusion Injury in a Skin Flap Mouse Model
}

\author{
Dong Kyun Rah', Hyung Jun Min², Yang Woo Kim², and Young Woo Cheon ${ }^{2 \bowtie}$ \\ 1. Department of Plastic and Reconstructive Surgery, Yonsei University, College of Medicine, Seoul, Republic of Korea \\ 2. Department of Plastic and Reconstructive Surgery, Gachon University Gil Medical Center, Incheon, Republic of Korea
}

$\triangle$ Corresponding author: Young Woo Cheon, M.D., Ph.D., Department of Plastic and Reconstructive Surgery, Gachon University Gil Medical Center, 21, Namdongdae-ro 774 beon-gil, Namdong-gu, Incheon, Republic of Korea. 405-760. Tel: +82-1577-2299 / Fax: +82-32-461-2774; E-mail: youngwooc@gmail.com

(C) Ivyspring International Publisher. This is an open access article distributed under the terms of the Creative Commons Attribution (CC BY-NC) license (https://creativecommons.org/licenses/by-nc/4.0/). See http://ivyspring.com/terms for full terms and conditions.

Received: 2017.02.08; Accepted: 2017.06.18; Published: 2017.07.19

\begin{abstract}
Background: Ischemia-reperfusion (I/R) injury is a leading cause of surgical skin flap compromise and organ dysfunction. Platelet-rich plasma (PRP) is an abundant reserve of various growth factors. Activated platelets play a role in endothelial damage during I/R injury; however, exogenous PRP could inhibit the production of reactive oxygen species. The goal of this study was to investigate the effect of PRP on I/R injury.

Methods: Four groups $(n=30)$ of C57BL/6N mice with lateral thoracic artery island flaps were used. Group A, the control group, received flap elevation and repositioning. Group B received PRP and repositioning. Group $C$ had 4 hours of ischemia and then were reperfused. Group $D$ received PRP, had 4 hours of ischemia, and then were reperfused. The survival area of flap tissue and blood perfusion were assessed. Histological evaluation included neutrophil counts. Reactive oxygen species and proinflammatory cytokines were measured to evaluate $I / R$ injury. Protein expression of phosphorylated apoptosis signaling regulating kinase-1 (pASK-1), P38MAPK, and PNF-KB was measured by western blot.

Results: PRP treatment enhanced the survival area and perfusion of the flap, reduced neutrophil accumulation in mice subjected to I/R injury. PRP treatment also showed a protective effect, with decreases in nitric oxide, myeloperoxidase, malondialdehyde concentrations. Additionally, PRP suppresses monocyte chemotactic protein-1, TNF- $\alpha$, IL-1 $\beta$, and IL-6. Finally, PRP decreased ASK-1 and NF-KB expression in tissues with I/R injury.

Conclusion: PRP acts as a protective factor during flap I/R injury by reducing reactive oxygen species level and proinflammatory cytokines via decreased expression of pASK-1 and pNF-kB.
\end{abstract}

Key words: Ischemia-reperfusion; Platelet-rich plasma; Axial flap

\section{Introduction}

Surgical skin flaps have been increasingly used in reconstructive surgery for the closure of various surgical defects. Partial or complete flap necrosis is a common problem after reconstructive flap surgery. An axial flap is preferred by surgeons because of its confidential pedicle; however, this flap is also more vulnerable to ischemia-reperfusion injury, or $\mathrm{I} / \mathrm{R}$ injury [1]. Management of the necrotizing flap usually needs time-consuming and repetitive dressing changes, or even a secondary surgical procedure [2].
Inadequate blood perfusion and $\mathrm{I} / \mathrm{R}$ injury are thought to be the major factors that cause several detrimental changes in the tissue and vasculature, resulting in flap necrosis [3]. Therefore, reducing I/ $\mathrm{R}$ injury in the necrotizing flaps has long been a clinical challenge. Such injuries are also common with organ transplantation surgeries.

$\mathrm{I} / \mathrm{R}$ injury is a complex process in which all steps of the inflammatory cascade may take part. Most of the damage is inflicted via leukocyte-endothelium 
interactions, reactive oxygen species, the complement cascade, mast cells, and immune complexes [4]. The role of molecular mediators has been shown by many studies [5]. Several efforts have been made to reduce I/R injury with small molecules, proteins, cytokines, and drugs [6], [7]. One possible way to prevent reactive oxygen species-mediated cellular injury is to augment endogenous oxidative defenses with dietary intake of antioxidants, such as vitamins A, C, or E [8]. Recently, attention has been focused on various non-vitamin antioxidants, such as phenolic compounds, which may also contribute to cellular antioxidative defense mechanisms and can be found in many plant species including green tea and edible fruits and vegetables [9]. However, these methods could not be translated to clinical applications because they have limited function and are expensive, complicated, and hard to handle.

Platelet-rich plasma (PRP) is an abundant reserve of various growth factors [10]. PRP can be collected autologously, and the cost of collection and processing is not expensive. Autologous PRP is biocompatible and safe, assuming no contamination occurs during processing. Therefore, for clinical use, no special considerations concerning antibody formation or risk of infection from donor are needed [11]. Many clinical devices are currently available to automatically prepare PRP [12]. Autologous PRP has been used intraoperatively for many years to clinically enhance wound healing and bone regeneration, reduce inflammation, and decrease blood loss in the fields of orthopedics and plastic surgery [13], [14]. Collectively, these studies provide strong evidence to support the clinical use of PRP in other settings, such as I/R injuries.

Despite these strengths, the evaluation of PRP quality remains controversial, and treatment with poor-quality PRP can result in injury [15]. Therefore, the definition of PRP was established by Marx et al. and is widely accepted in the field of regenerative medicine [16]. Because the scientific proof of bone and soft tissue healing enhancement has been shown using PRP with $1,000,000$ platelets $/ \mu \mathrm{l}$, it is this concentration of platelets in a $5-\mathrm{ml}$ volume of plasma which is the working definition of PRP today. [16]. The regenerative potential of PRP depends largely on the secretory cytokines released upon platelet activation, including vascular endothelial growth factor (VEGF), epidermal growth factor (EGF), platelet-derived growth factor (PDGF), transforming growth factor (TGF), and insulin-like growth factor (IGF)-1. The release of these cytokines occurs through platelet activation or physical disruption of the platelet-a-granule structure [17]. The most common method of PRP activation involves the addition of thrombin and calcium chloride $\left(\mathrm{CaCl}_{2}\right)$. Thrombin directly activates platelets, and the calcium ions from $\mathrm{CaCl}_{2}$ replenish those bound by acid citrate dextrose type A anticoagulant. Although this method is often used to activate PRP clinically, the activation that occurs during clot formation does not necessarily lead to complete release of growth factors [18]

Platelets are one of key component to modulate ischemia-reperfusion injury. There are some researches that platelets play a role to damage an endothelium during I/R injury with thrombosis [19], [20]. Therefore, the role of platelet in ischemia-reperfusion injury had been focused on enhancing tissue damage. However, exogenous PRP showed to reduce reactive oxygen species and mitochondrial depolarization in vitro. During myocardial ischemia-reperfusion, PRP could improve electrical and mechanical function of heart via altered mitochondrial function \& reduced apoptosis [21]. Also, there are studies that platelet-rich plasma could reduce I/ $\mathrm{R}$ injuries in the kidney and ovaries $[22,23]$. However, the protective effect of PRP to I/R injury in the flap model has not yet been revealed. The goal of this study was to investigate the effect of PRP on I/R injury in mouse axial pattern flap model. To discern the effects of PRP, we measured the survival of flap tissue, tissue perfusion of the flap, the production of reactive molecules, and proinflammatory cytokines.

\section{Materials and Methods}

\section{Preparation of platelet-rich plasma}

PRP was produced from full blood collects from 10-week-old C57BL/6N mice (Oriental bio, Seoul, Korea). An intracardiac blood volume of $1.2 \mathrm{ml}$ was obtained from the mice, mixed with $120 \mu \mathrm{l}$ of anticoagulant citrate dextrose solution formula A (ACDA), and mixed by inversion. It was centrifuged at $160 \times g$ for $15 \mathrm{~min}$ to separate the plasma (superior layer), red blood cells (inferior layer), and white blood cells (intermediate layer). Next, using a sterile syringe, the plasma and buffy coat were transferred to a new tube without anticoagulant and centrifuged for 10 min at $400 \times g$, yielding PRP with a mean concentration of 900,400 platelets $/ \mu 1$.

\section{Surgical procedure for the axial pattern flap model}

All animal experiments were conducted in accordance with the guidelines of the Korean animal protection statute and approved by the institutional review committee. C57BL/6N mice (Oriental bio, Seoul, Korea) of 8 weeks of age were used in this study. The animals were housed in a general, temperature- and humidity-controlled, pathogen-free 
environment on a cycle $12 \mathrm{~h}$ light/dark, and were allowed free access to food and water. After elevation of axial flap based on lateral thoracic artery, those mice that had irregular vessel anatomy were excluded for study. In total, 120 mice were used. They were placed randomly into four groups ( $n=30$ per group): group A - Control (flap elevation with PBS injection), group B - platelet-rich plasma, or PRP (flap elevation with PRP injection), group $\mathrm{C}$ - ischemia (flap elevation with 4 hours of clamping), and group D - ischemia and platelet-rich plasma, or ischemia-PRP (flap elevation with PRP injection, followed by 4 hours of clamping).

For the surgical procedure, mice were anesthetized by $30 \mathrm{mg} / \mathrm{kg}$ pentobarbital sodium by intraperitoneal injection after light anesthesia with isoflurane. After anesthesia, mice were placed on a heating pad to maintain constant body temperature throughout the surgery. After surgical cleansing of whole dorsal area, a dorsal lateral thoracic artery pedicled island skin flap (size: $1.5 \times 3.5 \mathrm{~cm}$ ) was raised in the caudal to cranial direction by careful dissection with direct visualization of pedicle. This island flap contained skin, subcutaneous tissue, and panniculus carnosus muscle. After flap elevation, a medical-grade silicon sheet was placed on the muscle bed as a barrier. In the control group (Group A), the flaps were injected with $120 \mu \mathrm{l}$ of PBS, inset to the original position and sutured using 4-0 polyglactin sutures without ischemia. In the PRP group (Group B), the flaps were subcutaneous injected with $120 \mu$ l of platelet-rich plasma. After injection, the flaps were inset to the original position and sutured without ischemia. In the ischemia group (Group C), the flap was elevated, $120 \mu \mathrm{l}$ of PBS was injected, and the pedicle was ligated with microclamp (Synovis, AL, USA) for $4 \mathrm{~h}$. After ischemia, the microclamp was removed to create a reperfusion injury [24]. The reperfusion of the flaps was checked using a laser doppler imager (Moor LDI2-HR, Moor Instruments, Axminster, UK) and the flaps were repositioned to the original position and sutured. In the ischemia-PRP group (Group D), the flap was elevated and subcutaneous injected with $120 \mu \mathrm{l}$ of platelet-rich plasma. After injection, the pedicle was ligated with microclamp for $4 \mathrm{~h}$, followed by removal of microclamp to create a reperfusion injury. The flaps were inset to the original position and sutured (Figure 1).

\section{Assessment of survival areas}

On days 1, 3, 5, 7, and 10 after the operation, the surviving area of the flap was measured by digital image analysis. Pictures of the flaps at the same distance were taken by a digital camera. (Nikon D70s; Nikon corporation, Tokyo, Japan) The surviving area of the flap was defined by two independent observers each three times, who examined the gross appearance, color, and consistency, elasticity, eschar, and the texture of the skin. The defined surviving area was measured using Image-Pro Plus Software (Media Cybernetics, MD, USA). The surviving areas were expressed as percentages of the total flap surface areas, as defined by the surgical borders.

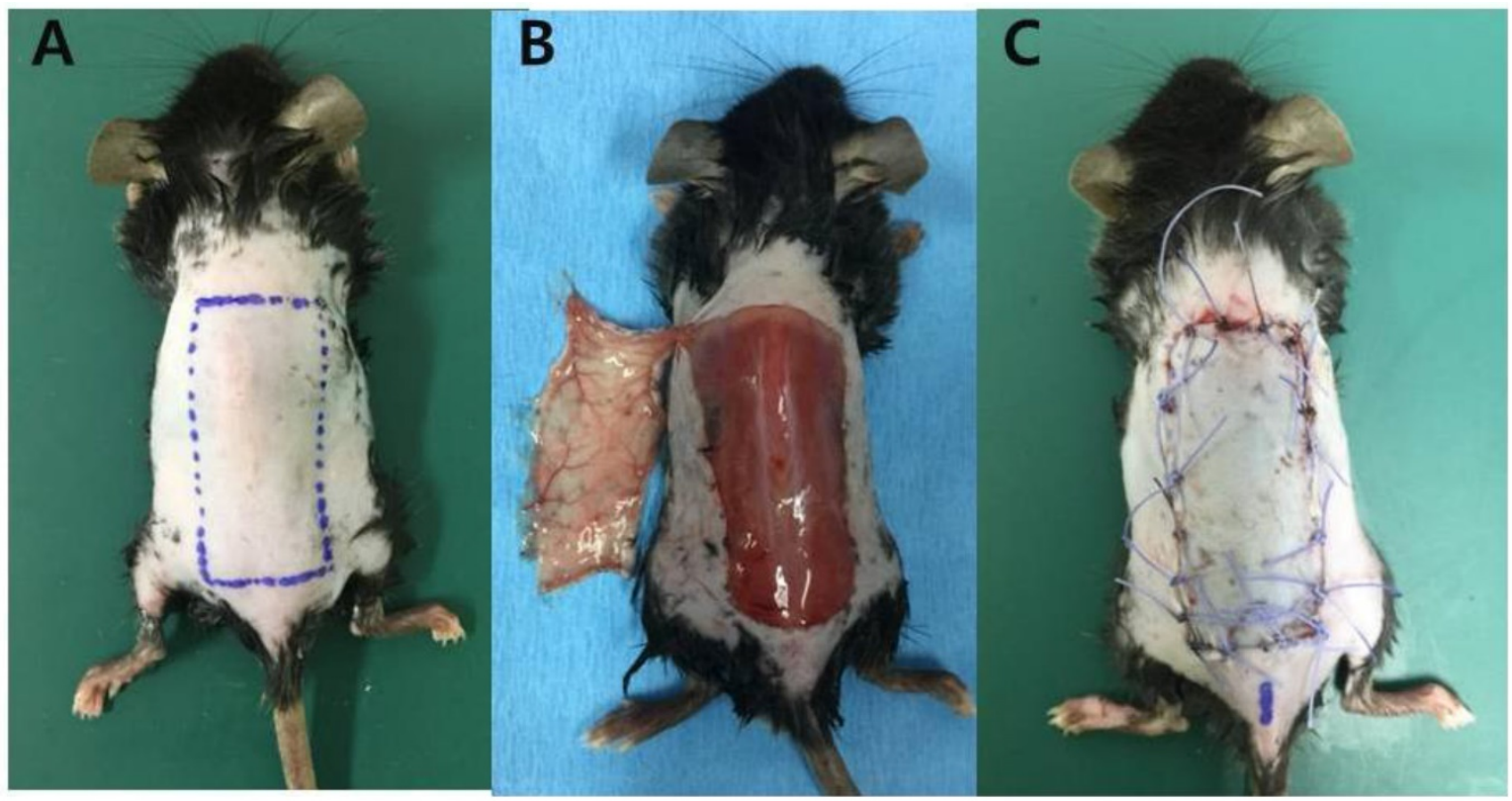

Figure 1. Surgical flap elevation procedure. (A) A design of the lateral thoracic artery-based axial island flap sized $1.5 \times 3.5 \mathrm{~cm}$ (B) The flap was elevated with preservation of left lateral thoracic artery. The pedicle was exposed at the undersurface of the flap. (C) The flap was returned to its original position with $4-0$ polyglactin sutures. 


\section{Hemodynamic assessment of the flaps}

In all mice from each group, tissue blood perfusion of the skin flap was measured with laser doppler flowmetry (Peri-Flux System 5000; Perimed, Inc., Stockholm, Sweden) on postoperative days 1, 3, 5,7 , and 10 . The probe was placed on the median line of the flap and the testing points were fixed on proximal, median, and distal portions, respectively. The room temperature was maintained at around $21^{\circ} \mathrm{C}$ during the blood flow measurements. For consistency, every measurement lasted at least 30 seconds. The results were expressed using the ratios of the postoperative blood perfusion units (BPU) to the preoperative BPU.

\section{Histopathological analysis}

Five mice per each group were euthanized to harvest the specimens. The full thickness specimens were taken from the center to the distal of each flap 12 hours after the onset of reperfusion. They were placed in $10 \%$ formalin and stained with Hematoxylin \& Eosin (H\&E) for histological examination to determine the infiltration of neutrophils to flap. Five-micrometer-thick sections were evaluated at $200 \times$ magnification, and the neutrophils per 75 random, non-overlapping fields were recorded. The mean number of neutrophils was calculated. Histological changes in H\&E-stained tissues were evaluated by analyzing tissue for indications of hyperemia, neutrophil aggregation, and intravascular microthrombosis.

\section{Measurement of ischemia-reperfusion injury}

Nitric oxide (NO), myeloperoxidase (MPO), malondialdehyde (MDA), and superoxide dismutase (SOD) were measure to evaluate I/ $R$ injuries [25]. For biochemical examination, $1 \times 1 \mathrm{~cm}$ sized specimens were taken from the center to the distal of the flaps, 12 $\mathrm{h}$ after reperfusion. Specimens were stored at $80^{\circ} \mathrm{C}$ immediately within individual containers. Because tissue nitrite $\left(\mathrm{NO}_{2}\right)$ and nitrate $\left(\mathrm{NO}_{3}\right)$ levels can be used to estimate NO production, we measured the concentration of these stable $\mathrm{NO}$ oxidative metabolites. Quantitation of $\mathrm{NO}_{2}$ and $\mathrm{NO}_{3}$ was based on the Griess reaction [25]. Results were expressed as $\mathrm{mmol} / \mathrm{g}$ tissue. Myeloperoxidase (MPO) was measured using the myeloperoxidase mouse ELISA kit (Abcam, Cambridge, UK). Samples were homogenized initially in $50 \mathrm{mmol} / \mathrm{L}$ potassium phosphate buffer and were centrifuged at $1,500 \times g$ for 10 minutes. In total, $500 \mu \mathrm{l}$ of homogenate was centrifuged at $40,000 \times g$ for 15 minutes at $4^{\circ} \mathrm{C}$. The supernatant was used for measuring proteins.

Malondialdehyde (MDA) was measured by measuring the presence of thiobarbituric acid reactive substances [26]. A total of $100 \mathrm{mg}$ per milliliter tissue was homogenized in buffer at a $\mathrm{pH}$ of 7.4. Artefactual production of additional MDA during processing was eliminated by the addition of $2 \%$ butylated hydroxytoluene to homogenized tissue. To this mixture, 20\% trichloroacetic acid in $0.6 \mathrm{~N}$ hydrochloride was added. The mixture was centrifuged at $10,000 \times g$ for 10 minutes at $4^{\circ} \mathrm{C}$. In total, $0.12 \mathrm{M}$ thiobarbituric acid in buffer $(\mathrm{pH}, 7.0)$ was added to the supernatant fraction. Pigment was measured spectrophotometrically at $532 \mathrm{~nm}$.

SOD enzyme-activity determination was based on the production of $\mathrm{H}_{2} \mathrm{O}_{2}$ from xanthine by xanthine oxidase and reduction of nitroblue tetrazolium. This measurement was made using a superoxide dismutase assay kit. (Abcam, Cambridge, UK) The product was evaluated spectrophotometrically. Results were expressed as $\mathrm{U} / \mathrm{ml}$.

\section{Real-time RT-PCR}

Flap samples were harvested in each group for analysis of proinflammatory cytokine expression during ischemia-reperfusion injury. Total RNA was harvested using TRIzol reagent (Invitrogen, Waltham, MA, USA) and was subjected to reverse transcription using a SuperScript III First-Strand Synthesis System for RT-PCR (Invitrogen) according to the manufacturer's instructions. Quantitative RT-PCR was performed with the SYBR system (Applied Biosystems, Foster City, CA, USA) using an ABI 7300 real-time PCR instrument (Life Technologies). SYBR probes and primers for monocyte chemotactic protein (MCP)-1, tumor necrosis factor (TNF)- $a$, Interleukin (IL)-1 $\beta$, IL-6, and $18 \mathrm{~S}$ were purchased from Takara Bio Inc. (Otsu, Japan). As an internal control, levels of $18 \mathrm{~S}$ were quantified in parallel with target genes. Normalization and fold changes were calculated using the comparative $\mathrm{Ct}$ method.

\section{Western blot}

Protein expression of all proteins, including apoptosis signaling regulating kinase 1 (ASK-1), p38MAPK, and nuclear factor-kappa B (NF-kB) p65, in the proximal portion of the skin flap was visualized by western blot. Total protein was extracted from the mouse skin flap using a RIPA lysis buffer (Thermo Scientific, Rockford, IL, USA) containing protease and phosphatase inhibitor cocktails (Roche, Indianapolis, USA) following the manufacturer's protocols.

The protein concentrations of extracts were determined using a BCA protein assay reagent kit (Pierce, Rockford, IL, USA). Then $30 \mu \mathrm{g}$ protein were loaded onto $12 \%$ SDS-PAGE gels. Proteins were resolved by electrophoresis and transferred onto PVDF membranes (Merck Millipore, Bedford, MA, 


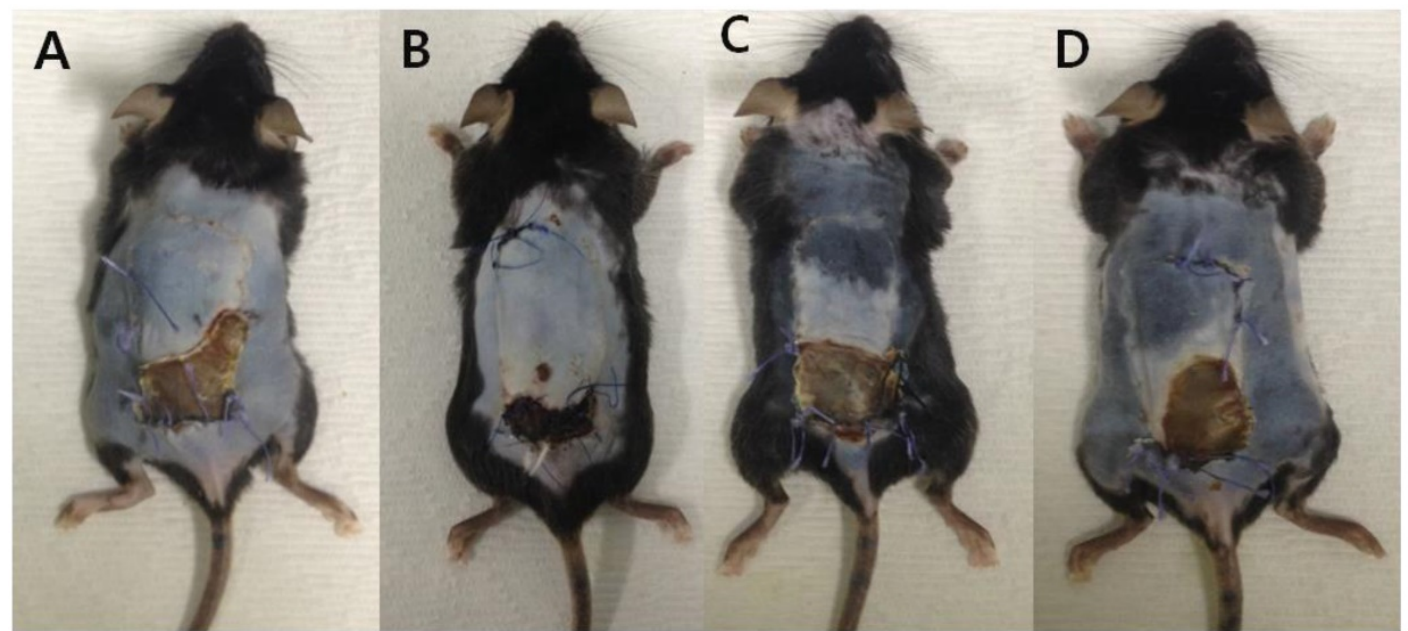

Figure 2. The flap survival area at POD\#10. (A) Control group, (B) platelet-rich plasma (PRP) group, (C) ischemia group, and (D) ischemia and PRP group. The ischemia-PRP group (Group D) exhibited a greater survival areas than the ischemia group (Group $C)(p<0.05)$.

USA). For immunoblotting, membranes were blocked with $5 \%$ nonfat dried milk in Tris-buffered saline for 1 $\mathrm{h}$. Blots were then incubated with primary antibodies specific for ASK-1, p38MAPK, phospho-p38MAPK, phospho-IкB (Cell Signaling technology, Danver, MA, USA), NF-kB (Abcam, Cambridge, UK), and $\beta$-actin (Cell Signaling technology, Danver, MA, USA, for an internal control) shaken overnight at $4^{\circ} \mathrm{C}$.

All primary antibodies were diluted 1:1000 in TBS, with the exception of $\beta$-actin, which was diluted 1:5000. After overnight incubation with primary antibody, the membranes were washed in Tris-buffered saline with $0.1 \%$ Tween 20 and incubated for $1 \mathrm{~h}$ with secondary anti-rabbit IgG peroxidase-conjugated antibody (Enzo Life Science, Farmingdale, NY, USA). The blots were developed with west-Zol PLUS kit (Intron biotechnology Co., Ltd. KOREA) Immunoreaction was visualized by chemiluminescence.

\section{Statistical analysis}

All data were expressed as the mean \pm standard deviation. The differences among groups were analyzed by one-way ANOVA. Post-hoc comparisons were done using Tukey test. Differences were regarded as statistically significant at $p$ values $<0.05$. All data were analyzed using $\mathrm{R}$ for Windows version 3.2. ( $\mathrm{R}$ foundation for Statistical computing, Vienna, Austria)

\section{Results}

\section{Survival areas of the flaps}

One day after the operation, we observed that the ischemia group (Group C) exhibited smaller survival areas than the other three groups. However, the difference was not statistically significant at this time. The differences in survival area became more robust after 3 days. The PRP group (Group B) and the ischemia-PRP group (Group D) exhibited larger survival areas than the other two groups. These differences were observed from postoperative day 3 through postoperative day $10(p<0.05)$. Mice in Group B had larger survival areas than those in group $\mathrm{D}$ at 10 postoperative days; however, this observation did not achieve statistical significance. The control group (Group A) showed larger survival area than Group $C$ with statistical significance $(p<0.05)$. The difference of survival area between groups $\mathrm{A}$ and $\mathrm{C}$ were initially detectable at postoperative day 5 , and the difference increased through postoperative day 10. For all groups, the decrease in survival area was most prominent from postoperative day 1 day to day 3 (Figures 2 and 3).

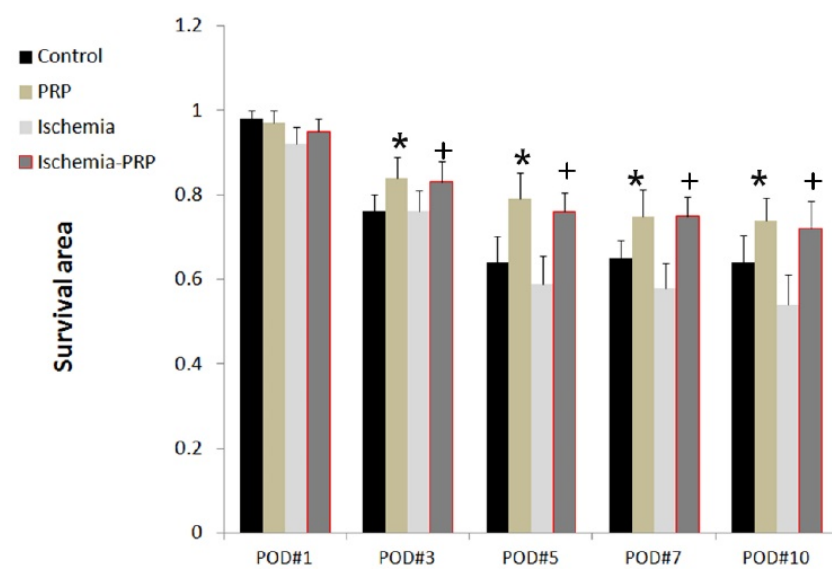

Figure 3. The flap survival area over 10 postoperative days. The PRP group (Group B) showed greater flap survival than the control group (Group A) from Day 3 to Day 10 postoperatively. Larger flap survival areas were observed in the ischemia-PRP group (Group D) than in the ischemia group (Group C). The differences increased after the $5^{\text {th }}$ postoperative day. The asterisk $(*)$ denotes statistical significance compared with the control group $(p<0.05)$. The dagger $(\dagger)$ denotes statistical significance compared with the ischemia group $(p<0.05)$ 


\section{Regional blood perfusion in the flaps}

We observed that all the flaps in the ischemia group (Group C) and the ischemia-PRP group (Group D) were cyanotic with adequate ischemia. After the removal of microclamp, the flaps showed hyperemic immediately, with apparent reperfusion injury. The regional blood perfusion (expressed as the ratio of postoperative to preoperative BPU) was different between the four groups when measured at postoperative day 1 (Figure 4). At this time, the PRP group (Group B) showed more blood perfusion than the other three groups; however, the difference was only statistically significant when compared with group C $(p<0.05)$. Group D exhibited more blood perfusion than Group $C$ with statistical significance $(p$ $<0.05$ ). At postoperative day 5, perfusion was significantly greater in the PRP group (Group B) than the control group (Group A). At this same timepoint, the perfusion in Group D was greater than in Group C with statistical significance $(p<0.05)$. Prior to postoperative day 5, Groups A and B did not show significant differences. We observed the greatest amount of perfusion in group $\mathrm{D}$ at postoperative day 10 , but this amount was not statistically significant from that of Group B. Group D displayed less perfusion than Group B on postoperative day 7; however, this trend reversed at postoperative day 10 (Figure 4).

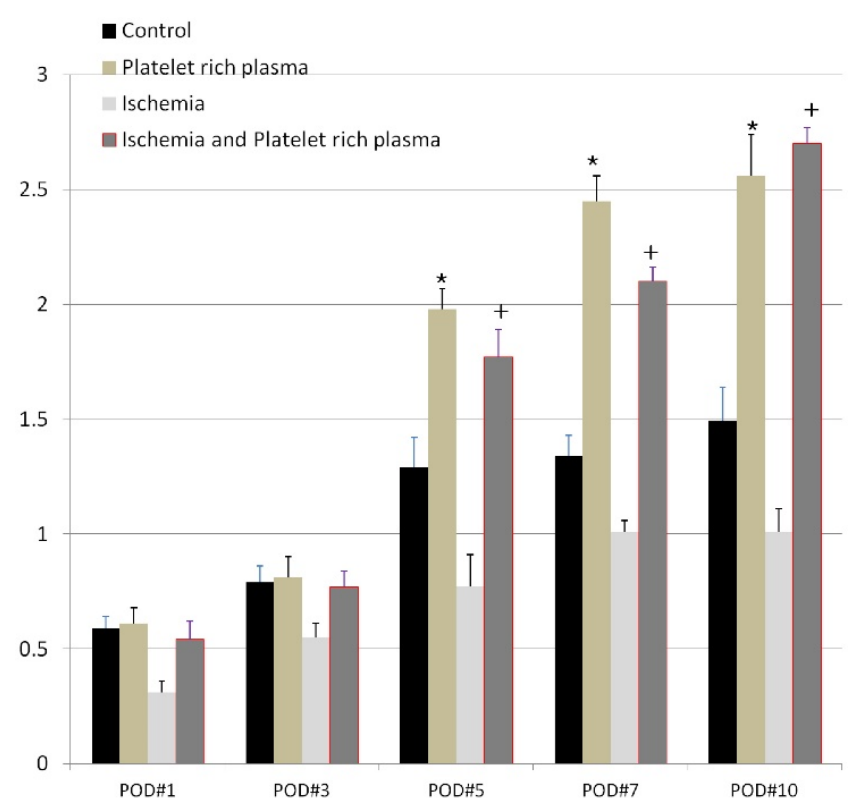

Figure 4. Flap perfusion as a function of postoperative day. The perfusion of each group increased over time postoperatively. At postoperative day 7 , the perfusion in the ischemia-PRP group (Group D) was greater than that in all other groups. However, the difference did not reach statistical significance between Groups B and D. The asterisk $(*)$ denotes statistical significance compared with the control (Group A) $(p<0.05)$. The dagger $(\dagger)$ denotes statistical significance compared with the ischemia group (Group C) $(p<0.05)$.

\section{Histopathological assessment}

We observed the highest neutrophil count in the ischemia group (Group C). However, PRP treatment reduced the neutrophil count by more than 2 -fold, even though $I / R$ injury. The ischemia-PRP group (Group D) had a larger neutrophil count than the control group (Group A) and the PRP group (Group B). Non-I/R injury groups (Groups A and B) had lower neutrophil counts than I/R injury groups (Groups C and D; Figure 5A and 5B). Group C showed extensive hyperemia, neutrophil aggregations, and intravascular microthrombus visualize by $H \& E$ stain. However, we observed less neutrophil aggregation in stained tissues from Group D (Figure 5A).

\section{Measurement of ischemia-reperfusion injury}

To evaluate the level of $I / R$ injury in the axial flap, we measured levels of nitric oxide (NO), myeloperoxidase (MPO), malondialdehyde (MDA), and superoxide dismutase (SOD). NO level was highest in the ischemia group (Group $C ; p<0.05$ ). The control group (Group A) and the PRP group (Group B) exhibited lower levels of NO than Group C; however, the difference between Groups A and B was not statistically significant. The ischemia-PRP group (Group D) showed significantly lower levels of NO compared with Group C. When comparing Groups A and $\mathrm{B}$, we found that PRP could not affect production of these compounds without tissue exposure to ischemic conditions. However, PRP could reduce reperfusion injury effectively after 4 hours of ischemia (Figure 6A).

Tissue concentrations of MPO in Group C were significantly higher than those in the other three groups (Figure 6B). Groups A and B displayed significantly lower levels of MPO than Groups $C$ and $\mathrm{D}(p<0.05)$. This effect was not observed without exposure to ischemia.The tissue concentration of MDA in Group D was greater than those in Groups A and B. However, the level of MDA in Group D was significantly lower than that in Group C. The non-I/R injury groups exhibited lower levels of MDA than the two I/R injury groups with statistical significance (Figure 6C). In group C, SOD levels were lower than those in the other three groups $(p<0.05$ However, SOD levels were higher in Group D than in Group C (Figure 6D).

\section{Proinflammatory cytokines}

We observed that PRP suppressed mRNA levels of proinflammatory cytokines and chemokines. The tissue samples were harvested at postoperative day 1 . In the ischemia-PRP group (Group D), the level of Ccl2 (which encodes Mcp-1) was significantly lower 
than that in the ischemia group (Group C). Interestingly, the level of $\mathrm{Ccl} 2$ in Group D did not show a statistically significant difference relative to the control group (Group A) or the PRP group (Group B). The expression levels of Tnf, Il1b, and $I l 6$ were significantly decreased in Group D compared with Group C. However, the expression levels of Tnf, Il1b, Il6 in non-I/R injury groups were generally lower than those for I/R injury groups (Figure 7A-D).

\section{Signaling pathways}

The expression of phospho-apoptosis signalregulating kinase-1 (pASK-1), phospho-p38MAPK, and phosphor-NF-kB p65 was evaluated by western blot after 12 hours of reperfusion. The ischemia group (Group C) showed that I/R injury increased phosphorylation of ASK-1, p38, and NF-KB. In the ischemia-PRP group (Group D), PRP significantly reduced pASK-1 expression, indicating that PRP could protect flaps from I/R injury by suppressing ASK-1 (Figure 8A and B). Although phosphop38MAPK was slightly decreased in Group D, this difference was not statistically significant $(p>0.05)$. The PRP group (Group B) exhibited a slight decrease in phospho-p38 compared with the control group (Group A); however, this difference was not
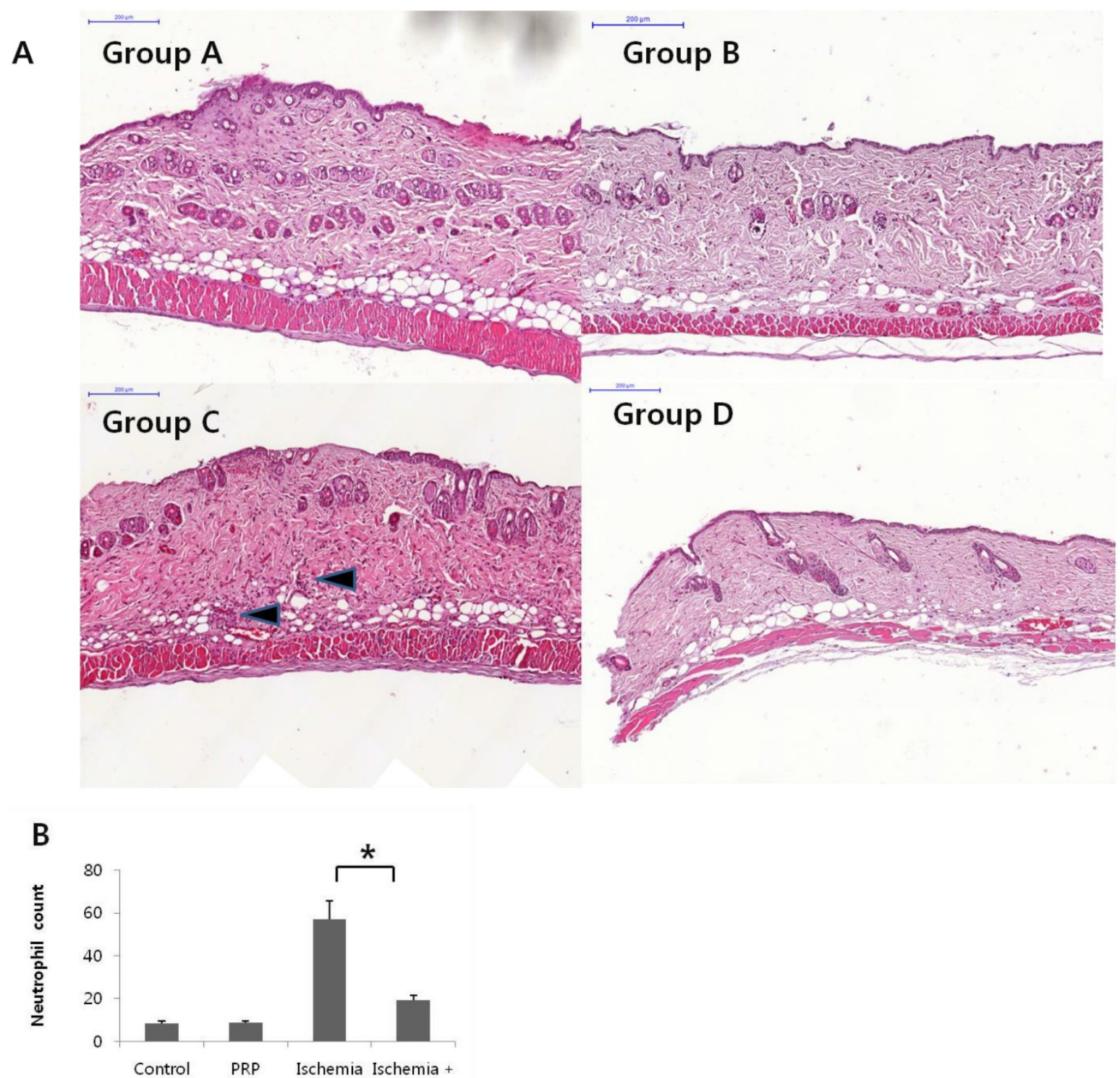

PRP

Figure 5. (A) Histopathological analysis of tissue damage in mouse skin flaps. Hematoxylin and eosin-stained tissue examined at 200x magnification showed extensive hyperemia, neutrophil aggregation, and intravascular microthrombi in the ischemia group (Group C). In the control group (Group A) and the PRP group (Group B), neutrophil aggregation and microthrombi could not be found. In the ischemia-PRP group (Group D), minimal neutrophil infiltration was observed. Much more neutrophil infiltration occurred in Group $C$ (black arrow); however, PRP treatment decreased the neutrophil count after ischemia. Arrow indicates neutrophil infiltration. (B) Neutrophil count of flap specimens. Groups $A$ and $B$ showed lower neutrophil counts than I/R injury groups (Groups $C$ and $D$ ). An increase of $>5$-fold was observed in group $C$ compared with non-I/R injury groups. However, PRP reduced the neutrophil count with statistical significance. 
statistically significant. Non-I/R injury groups showed lower levels of phospho-p38 than I/R injury groups. (Figure $8 \mathrm{C}$ and $8 \mathrm{D}$ ). I/ $\mathrm{R}$ injury increased the activity of pNF-kB in Group C. However, PRP treatment reduced the expression of $\mathrm{pNF}-\mathrm{kB}$ in Group $\mathrm{D}$, displaying apparent degradation of IKB (Figure 8E and $8 \mathrm{~F}$ ).
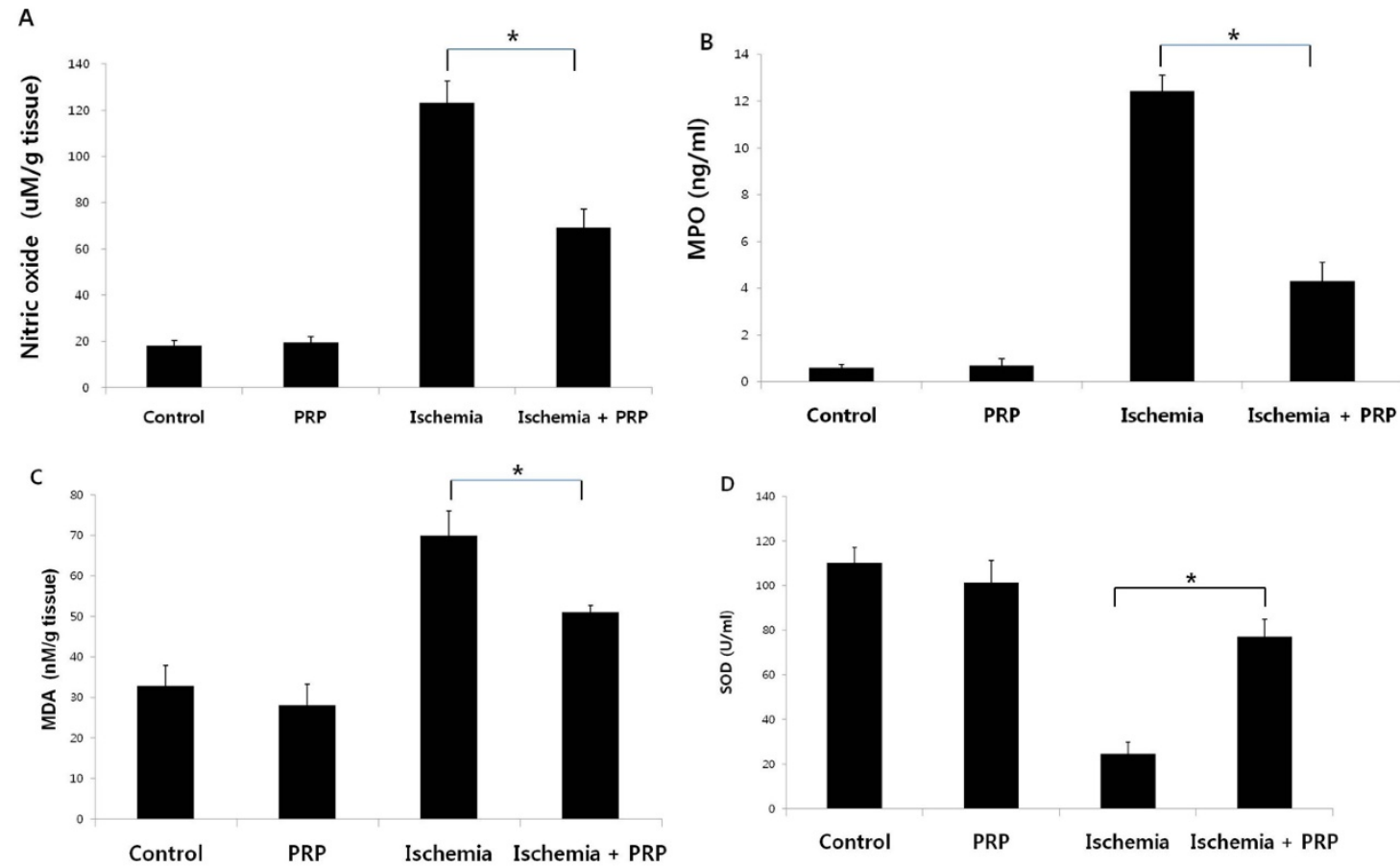

Figure 6. Measurements of nitric oxide (NO), myeloperoxidase (MPO), malondialdehyde (MDA), and superoxide dismutase (SDO). (A) NO level was highest in the ischemia group (Group $C$ ) than other three groups $(p<0.05)$. The ischemia-PRP group (Group D) showed a significantly lower level of NO than Group C, but this level was higher than those for the control group (Group A) and the PRP group (Group B). PRP alone does not reduce reperfusion injury; however PRP treatment decreased I/R injury after 4 hours of ischemia. (B) The tissue MPO concentration in group $C$ was significantly higher than that in the other three groups. PRP decreased MPO concentrations when combined with exposure to ischemia (Group D). (C) Tissue MDA concentration in Group D was higher than those in Groups A and B; but significantly lower than Group C. The two groups without ischemia exposure exhibited lower levels of MDA than the two I/R injury groups with statistical significance. (D) We observed higher SOD levels in Groups A and B than in Group D; however, Group D showed higher levels of SOD relative to Group C. $(p<0.05)$
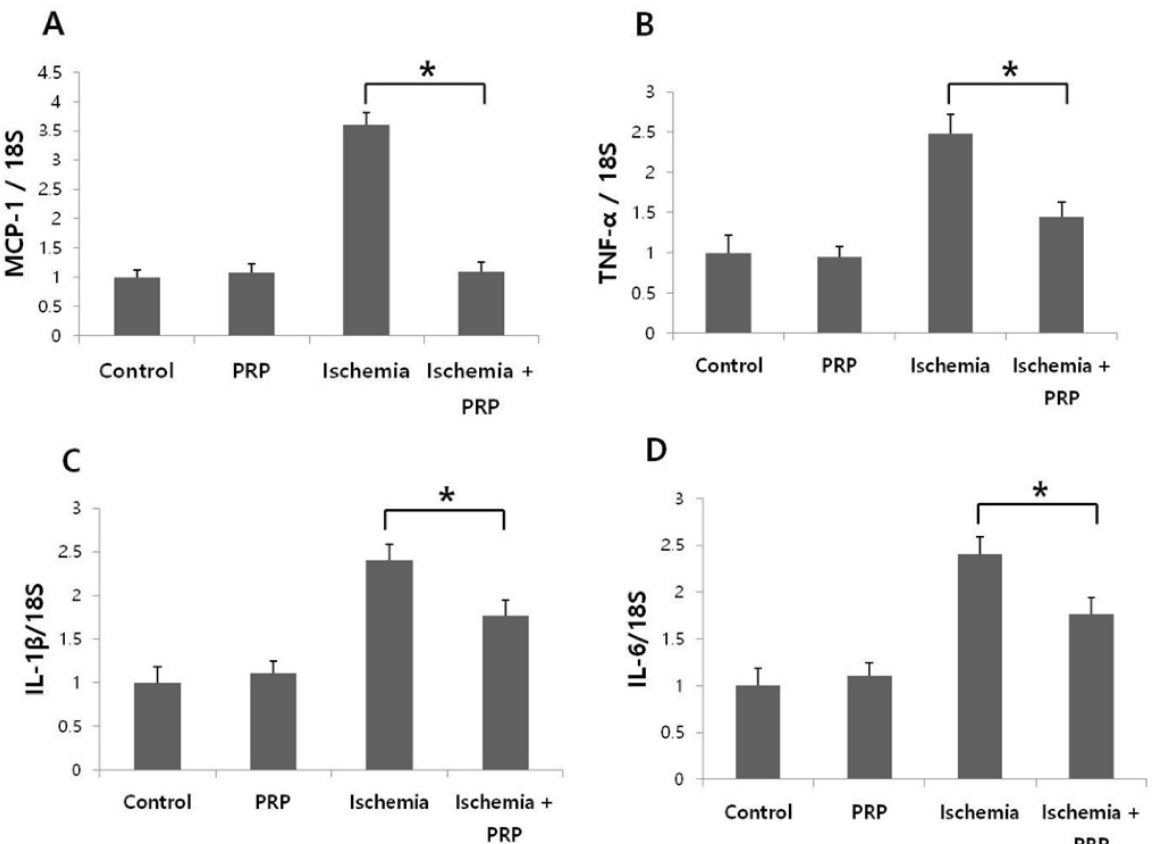

PRP

Figure 7. mRNA levels of monocyte chemotactic protein-1 (Ccl2), Tnf, IIlb, Il6, mRNA levels. To calculate mRNA expression, control mice (Group A) were assigned values of 1. (A) The ischemia-PRP group (Group D) showed decreased expression of $C c 12$ relative to the ischemia group (Group C; $p<0.05$ ). The level of $C c 12$ between Groups $A$, B, D did not show statistical differences. (B) The expression of Tnf was higher in Group $C$ than Group D. Non-l/R injury groups showed lower levels of expression than Group D ( $P$ $<0.05$ ). (C) Groups A and B did not show any statistically significant differences in $I I l b$ expression. PRP significantly suppressed the expression of $I I I b$ compared to group C. ( $P$ $<0.05$ ) (D) 116 expression in Group $C$ was higher than non-I/R injury groups. However, PRP reduced II6 expression in Group D ( $p<0.05)$. 


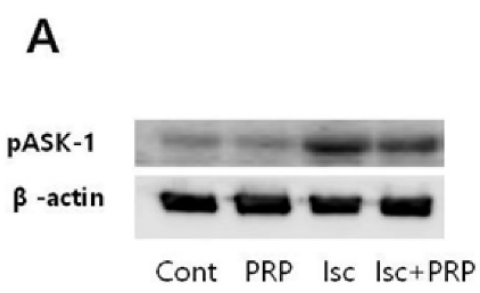

C

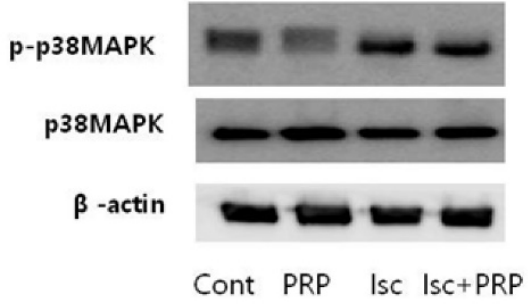

E

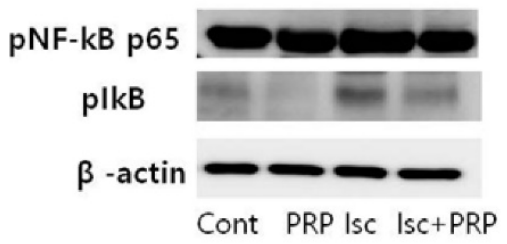

B

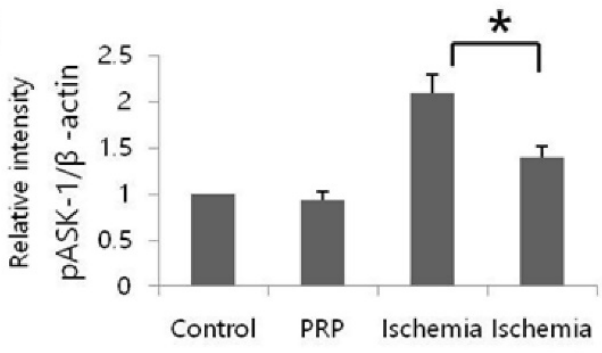

D

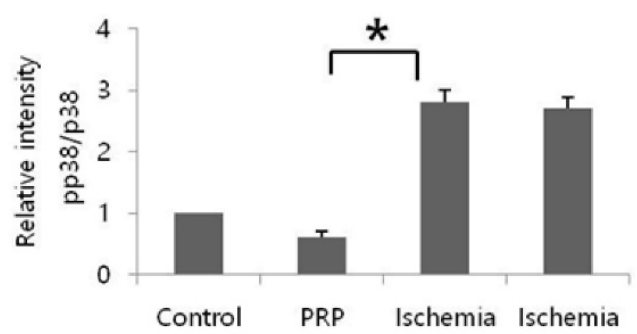

F

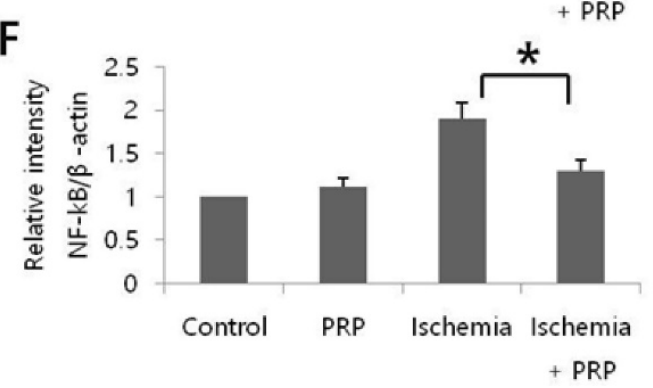

Figure 8. Relative expression of ASK-1 and p38MAPK. Expression levels of phosphorylated apoptosis signal-regulating kinase 1 and p38 were detected by western blot analysis in skin flaps after reperfusion for 12 hours. (A) Representative blot of PASK-1. (B) Quantification of protein levels of $p A S K-1$. I/R injury increased $p A S K-1$ expression; however, platelet-rich plasma significantly reduced pASK-1, ${ }^{*} p<0.05$. (C) Representative blotting of $p 38$. (D) Quantification of protein levels of $p 38$. Groups $C$ and D showed more expression of phospho-p38 expression than groups $A$ and $B$. The difference between groups $C$ and $D$ was not significant. Non-I/R injury groups showed lower levels of phospho-p38 than I/R injury groups. ${ }^{*} p<0.05$. (E) Representive blotting of $\mathrm{pNF}-\mathrm{kB}$. (F) Quantification of protein levels of $\mathrm{pNF}-\mathrm{kB}$. PRP reduced the expression of $\mathrm{pNF-kB}$ in group D compared to group C. $*_{p}<0.05$.

\section{Discussion}

This study was designed to investigate the role of PRP during I/R injury in a dorsal pedicled flap mouse model. I/R injury is inevitable during microsurgical free flap transfer, organ transplantation, and other major surgeries [27]. This injury can lead to organ compromise or even permanent dysfunction. Thus, it is very important to clarify the mechanisms of $I / R$ injury and search for effective treatments to prevent it. In this study, we determined that PRP significantly increased the survival area of the flap, regardless of whether the flap experiences an I/R injury. Interestingly, the survival area of in the ischemia-PRP group was greater than the control group, which did not undergo an I/R injury (Figure 1). Also, the difference between the ischemia group and the ischemia-PRP group was greater than that between the control group and the PRP group. This phenomenon may occur because prolonged ischemia can open choke vessels and increase angiogenesis [28]. In this way, ischemia itself could be beneficial to flap surgery by increasing angiogenesis [29]. We confirmed that more perfusion occurred in the ischemia-PRP group at the end of the study. In contrast, the ischemia group had the lowest perfusion of the four groups (Figure 2). While ischemia has the beneficial effect of stimulating angiogenesis, reperfusion induces tissue damage and apoptosis [30]. Therefore, in terms of free flap surgery and organ transplantation, minimizing the reperfusion injury is essential to preserving the tissue.

PRP has been used in various clinical applications, including periodontal and oral surgery, maxillofacial and aesthetic plastic surgery, spinal fusion, cardiac bypass surgery, and treatment of soft tissue ulcers [15], [31]. PRP administered during surgical procedures under sterile conditions is easily performed and safe to use. Moreover, PRP lacks surface immunogenic antigens, and thus allergic reactions are not of great concern. The secreted growth factors induced by PRP immediately bind to the external surface of membranes of cells in the graft, 
flap, or wound via transmembrane receptors [18]. Recently, PRP has been reported to activate the antioxidant response element in a tenocyte culture model through the Nrf3-ARE pathway in a dose-dependent manner [32].

Some procedures improve skin flap survival after an I/R injury. These include procedures that restore high levels of energy-rich phosphate compounds (e.g. ATP- $\mathrm{MgCl}_{2}$ ) or maintain the oxygen supply (e.g., synthetic hemoglobin substrate Fluosol-DA, together with a thromboxane synthetase inhibitor such as dazoxiben hydrochloride or UK-38) [25], [33], [34]. However, these procedures are expensive, difficult to use, and require clinical trials to increase the number of indications. PRP is easy to use, cheap, readily made, and stable without host rejection when used as autologous manner. In this study, PRP increased survival of $I / R$ injured flaps. We thought that this improvement may have resulted from its anti-inflammatory properties and its ability to decrease NF- $\mathrm{kB}$ activity, providing protective effects against I/R injury.

Here, we show that levels of $\mathrm{MPO}, \mathrm{NO}$, and MDA levels were decreased in the ischemia-PRP group. On the contrary, SOD enzyme activities were increased in the ischemia-PRP group relative to the ischemia group (Figure 6). MPO is a characteristic constituent of neutrophil granules, and it is used as a biochemical marker for tissue invasion of neutrophils [24]. Preventing or decreasing neutrophil invasion to reperfused tissues by blocking any step of neutrophil activation has been shown to decrease tissue MPO activity [35]. The decreased SOD activity and increased MPO, NO and MDA content in the ischemia group demonstrate that redox imbalance and high levels of reactive oxygen species occur in I/ $R$ injured flaps. However, PRP treatment provided a protective effect against $\mathrm{I} / \mathrm{R}$ injury by increasing SOD levels. MDA is an end product of lipid peroxidation and a known indicator of tissue injury. Interestingly, we observed low MDA levels in both non-ischemic groups. In this study, MDA levels were effectively suppressed by PRP in I/R injured tissues; however, PRP treatment did not reduce the MDA level in the non-I/R injury group.

Improved tissue survival was accompanied by decreased neutrophil recruitment, tissue lipid oxidation, and inflammatory cytokine levels (Figure 5 and 7). These findings indicate that PRP treatment reduces the inflammatory response. Macrophages infiltrate the tissues during the early phase of the response to ischemia-reperfusion injury and are involved in inflammation by secreting proinflammatory mediators, including Mcp-1, NO, Il-1, and Il-6 [36]. We found that PRP effectively suppresses expression of inflammatory cytokines, such as Ccl2, Tnf, Illb, and Il6. Interestingly, PRP treatment reduced $\mathrm{Ccl} 2$ levels to those of the non-I/R injury groups (Figure 5A). The chemotactic activity of Mcp-1 induces diapedesis of monocytes from the lumen to the subendothelial space. Once there, monocytes become foam cells and initiate fatty streak formation, which leads to atherosclerotic plaque formation. Inflammatory macrophages probably play a role in plaque rupture and the resulting ischemic episode, as well as restenosis after angioplasty. There is strong evidence that Mcp-1 plays a major role in myocarditis, I/ R injury in the heart, and transplant rejection [37]. We posit that the protective effect of PRP treatment against I/ $R$ injury might be associated with both suppression of inflammation and promotion of angiogenesis [38]

Apoptosis is an important and primary form of cell death during skin flap $I / R$ injury. The reactive oxygen species produced during early reperfusion are known to activate apoptosis cascades [27]. Here, PRP treatment reduced reactive oxygen species concentrations. In addition to its direct antioxidant action, PRP treatment also acts as an apoptosis regulatory messenger, which we observed while investigating phospho-ASK-1 expression. ASK-1 is a typical member of the mitogen-activated protein kinase kinase kinase (MAPKKK) family, and a critical signaling component in the progression of reactive oxygen species-induced apoptosis [39]. In this study, the expression level of phospho-ASK-1 was higher in $I / R$ injury groups than in non-I/ $R$ injury groups (Figure 6). However, PRP reduced the expression of ASK-1 in the ischemia-PRP group. In the contrast, p-p38 was unaffected by PRP. NF-kB activation has been demonstrated in many articles investigating I/ $R$ injuries [1]. The activation of NF-kB leads to inflammation, followed by degradation of ІкB [4]. We confirmed that PRP could reduce the activation of NF- $\mathrm{kB}$ during I/R injury, and this reduction of inflammation could be one more molecular contributor to flap survival.

The limitation of this study is the use of mice with allogenic PRP. PRP was used in autologous fashion in clinical situation. However, we used allogenic PRP from other mouse because proper amount of blood was necessary to obtain adequate amount of PRP. The further investigation was crucial to reveal protective effect of PRP against I/R injury in human and the adequate dosage in clinical usage.

\section{Conclusion}

In this study, we investigated the effect of PRP on I/R injuries using the axial pattern flap model. Our results demonstrate that PRP acts as a protective 
factor during I/ $\mathrm{R}$ injury by reducing reactive oxygen species levels and proinflammatory cytokines. This effect could be mediated by suppressing ASK-1 and pNF-kB pathways.

\section{Acknowledgement}

This research was supported by Basic Science Research Program through the National Research Foundation of Korea(NRF) funded by the Ministry of Science, ICT \& Future Planning(NRF-2014R1A1A1037258); a research focused grant from Gil Hospital (FRD 2013-41).

\section{Competing Interests}

The authors declare that no potential conflict of interest relevant to this article exist.

\section{References}

1. Coskunfirat OK, Cinpolat A, Bektas G, Ogan O, Taner T. Comparing different postconditioning cycles after ischemia reperfusion injury in the rat skin flap. Annals of plastic surgery. 2014; 72: 104-7.

2. Kim H, Kawazoe T, Han D, Matsumara K, Suzuki S, Tsutsumi S, et al. Enhanced wound healing by an epigallocatechin gallate-incorporated collagen sponge in diabetic mice. Wound repair and regeneration. 2008; 16: 714-20.

3. Tatlidede SH, Murphy AD, McCormack MC, Nguyen JT, Eberlin KR, Randolph MA, et al. Improved survival of murine island skin flaps by prevention of reperfusion injury. Plastic and reconstructive surgery. 2009; 123: 1431-9.

4. Kim HJ, Xu L, Chang KC, Shin SC, Chung JI, Kang D, et al. Anti-inflammatory effects of anthocyanins from black soybean seed coat on the keratinocytes and ischemia-reperfusion injury in rat skin flaps. Microsurgery. 2012; 32: 563-70.

5. Klein MB, Chan PH, Chang J. Protective effects of superoxide dismutase against ischemia-reperfusion injury: development and application of a transgenic animal model. Plastic and reconstructive surgery. 2003; 111: 251-5.

6. Cordeiro PG, Mastorakos DP, Hu QY, Kirschner RE. The protective effect of L-arginine on ischemia-reperfusion injury in rat skin flaps. Plastic and reconstructive surgery. 1997; 100: 1227-33.

7. Gideroglu K, Yilmaz F, Aksoy F, Bugdayci G, Saglam I, Yimaz F. Montelukast protects axial pattern rat skin flaps against ischemia/reperfusion injury. The Journal of surgical research. 2009; 157: 181-6.

8. Zhu Z, Zhu J, Zhao X, Yang K, Lu L, Zhang F, et al. All-Trans Retinoic Acid Ameliorates Myocardial Ischemia/Reperfusion Injury by Reducing Cardiomyocyte Apoptosis. PLoS One. 2015; 10: e0133414-e.

9. Valcic S, Burr JA, Timmermann BN, Liebler DC. Antioxidant chemistry of green tea catechins. New oxidation products of (-)-epigallocatechin gallate and (-)-epigallocatechin from their reactions with peroxyl radicals. Chemical research in toxicology. 2000; 13: 801-10.

10. Fréchette JP, Martineau I, Gagnon G. Platelet-rich plasmas: growth factor content and roles in wound healing. Journal of dental research. 2005; 84: 434-9.

11. Li W, Enomoto M, Ukegawa M, Hirai T, Sotome S, Wakabayashi Y, et al. Subcutaneous injections of platelet-rich plasma into skin flaps modulate proangiogenic gene expression and improve survival rates. Plastic and reconstructive surgery. 2012; 129: 858-66.

12. Everts PA, Brown Mahoney C, Hoffmann JJ, Schönberger JP, Box HA, van-Zundert $A$, et al. Platelet-rich plasma preparation using three devices: implications for platelet activation and platelet growth factor release. Growth factors. 2006; 24: 165-71.

13. Getgood A, Henson F, Brooks R, Fortier LA, Rushton N. Platelet-rich plasma activation in combination with biphasic osteochondral scaffolds-conditions for maximal growth factor production. Knee surgery, sports traumatology, arthroscopy. 2011; 19: 1942-7.

14. Borrione P, Di Gianfrancesco A, Pereira MT, Pigozzi F. Platelet-rich plasma in muscle healing. American journal of physical medicine \& rehabilitation. 2010; 89: 854-61.

15. Marx RE. Platelet-rich plasma: evidence to support its use. Journal of oral and maxillofacial surgery. 2004; 62: 489-96.

16. Marx RE. Platelet-rich plasma (PRP): what is PRP and what is not PRP? Implant dentistry. 2001; 10: 225-8.

17. Eppley BL, Woodell JE, Higgins J. Platelet quantification and growth factor analysis from platelet-rich plasma: implications for wound healing. Plastic and reconstructive surgery. 2004; 114: 1502-8.

18. Oh DS, Cheon YW, Jeon YR, Lew DH. Activated platelet-rich plasma improves fat graft survival in nude mice: a pilot study. Dermatologic surgery. 2011; 37: 619-25.
19. Rodrigues SF, Granger DN. Role of blood cells in ischaemia-reperfusion induced endothelial barrier failure. Cardiovascular research. 2010; 87: 291-9.

20. Tamura T, Kondo T, Pak S, Nakano Y, Murata S, Fukunaga K, et al. Interaction between Kupffer cells and platelets in the early period of hepatic ischemia-reperfusion injury--an in vivo study. The Journal of surgical research. 2012; 178: 443-51.

21. Hargrave B, Li F. Nanosecond pulse electric field activation of platelet-rich plasma reduces myocardial infarct size and improves left ventricular mechanical function in the rabbit heart. The Journal of extra-corporeal technology. 2012; 44: 198-204.

22. Martín Solé O, Rodó J, García Aparicio L, Blanch J, Cusí V, Albert A. Effects of Platelet-Rich Plasma (PRP) on a Model of Renal Ischemia-Reperfusion in Rats. PLoS One. 2016; 11: e0160703-e.

23. Bakacak M, Bostanci MS, İnanc F, Yaylali A, Serin S, Attar R, et al. Protective Effect of Platelet Rich Plasma on Experimental Ischemia/ Reperfusion Injury in Rat Ovary. Gynecologic and obstetric investigation. 2016; 81: 225-31.

24. Cetin C, Köse AA, Aral E, Colak O, Erçel C, Karabağli Y, et al. Protective effect of fucoidin (a neutrophil rolling inhibitor) on ischemia reperfusion injury: experimental study in rat epigastric island flaps. Annals of plastic surgery. 2001; 47: 540-6.

25. Aydogan H, Gurlek A, Parlakpinar H, Askar I, Bay Karabulut A, Aydogan N, et al. Beneficial effects of caffeic acid phenethyl ester (CAPE) on the ischaemia-reperfusion injury in rat skin flaps. Journal of plastic, reconstructive \& aesthetic surgery. 2007; 60: 563-8.

26. Parlakpinar H, Ozer MK, Sahna E, Vardi N, Cigremis Y, Acet A. Amikacin-induced acute renal injury in rats: protective role of melatonin. Journal of pineal research. 2003; 35: 85-90.

27. Yin Z, Ren H, Liu L, Chen W, Gan C, Jiao H, et al. Thioredoxin Protects Skin Flaps from Ischemia-Reperfusion Injury: A Novel Prognostic and Therapeutic Target. Plastic and reconstructive surgery. 2016; 137: 511-21.

28. Williams BA, Currie RW, Morris SF. Impact of arteriogenesis in plastic surgery: choke vessel growth proceeds via arteriogenic mechanisms in the rat dorsal island skin flap. Microcirculation. 2009; 16: 235-50.

29. O'Toole G, MacKenzie D, Buckley MF, Lindeman R, Poole M. A review of therapeutic angiogenesis and consideration of its potential applications to plastic and reconstructive surgery. British journal of plastic surgery. 2001; 54: $1-7$.

30. Wu C, Bratton SB. Regulation of the intrinsic apoptosis pathway by reactive oxygen species. Antioxidants \& redox signalling. 2013; 19: 546-58.

31. Eppley BL, Pietrzak WS, Blanton M. Platelet-rich plasma: a review of biology and applications in plastic surgery. Plastic and reconstructive surgery. 2006; 118: 147-59e.

32. Tohidnezhad M, Varoga D, Wruck CJ, Brandenburg LO, Seekamp A, Shakibaei $\mathrm{M}$, et al. Platelet-released growth factors can accelerate tenocyte proliferation and activate the anti-oxidant response element. Histochemistry and cell biology. 2011; 135: 453-60

33. Reiter RJ, Tan D. Melatonin: a novel protective agent against oxidative injury of the ischemic/reperfused heart. Cardiovascular research. 2003; 58: 10-9.

34. Birincioglu M, Aksoy T, Olmez E, Acet A. Protective effect of ACE inhibitors on ischemia-reperfusion-induced arrhythmias in rats: is this effect related to the free radical scavenging action of these drugs? Free radical research. 1997; 27: 389-96.

35. Stotland MA, Kerrigan CL. E- and L-selectin adhesion molecules in musculocutaneous flap reperfusion injury. Plastic and reconstructive surgery. 1997; 99: 2010-20.

36. Uchiyama A, Yamada K, Perera B, Ogino S, Yokoyama Y, Takeuchi Y, et al. Protective effect of MFG-E8 after cutaneous ischemia-reperfusion injury. Journal of investigative dermatology. 2015; 135: 1157-65.

37. Niu J, Kolattukudy PE. Role of MCP-1 in cardiovascular disease: molecular mechanisms and clinical implications. Clinical science. 2009; 117: 95-109.

38. Sonmez TT, Vinoqradov A, Zor F, Kweider N. The effect of platelet rich plasma on angiogenesis in ischemic flaps in VEGFR2-luc mice. Biomaterials. 2013; 34:2674-82

39. Matsukawa J, Matsuzawa A, Takeda K, Ichijo H. The ASK1-MAP kinase cascades in mammalian stress response. The Journal of biochemistry. 2004; 136: $261-5$. 